Mathematical Research Letters 4, 23-33 (1997)

\title{
DISTINGUISHING ISOSPECTRAL NILMANIFOLDS BY BUNDLE LAPLACIANS
}

\author{
Carolyn Gordon, He Ouyang, and Dorothee Schueth
}

\section{Introduction}

Two closed Riemannian manifolds are said to be isospectral if the associated Laplace Beltrami operators, acting on smooth functions, have the same eigenvalue spectrum. When two metrics are isospectral in this sense, one may ask whether the metrics can nonetheless be distinguished by additional spectral data - e.g., by the spectra of the Laplacians acting on $p$-forms.

In this article we consider isospectral deformations, i.e., continuous families of isospectral metrics on a given manifold. In 1984, E. N. Wilson and the first author $[\mathrm{GW}]$ gave a method for constructing isospectral deformations $\left(M, g_{t}\right)$ on nilmanifolds $M$, generalized slightly in ([Go], [DG2]). The construction of these isospectral families is reviewed in section one. These were the only known examples of isospectral deformations of metrics on closed manifolds until recently when R. Gornet [Gt] constructed new examples of isospectral deformations of metrics on nilmanifolds by a different method. However, the metrics in Gornet's examples can be distinguished by the spectra of the associated Laplacians acting on 1-forms. Thus we focus here on the earlier deformations.

The metrics in these deformations are actually strongly isospectral; i.e., all natural self-adjoint elliptic partial differential operators associated with the metrics are isospectral. (Here natural refers to operators that are preserved by isometries; for the definition, see [St].) We show in section two, answering a question raised to us by P. Gilkey, that they are even strongly $\pi_{1}$-isospectral; i.e., all natural self-adjoint elliptic operators with coefficients in locally flat bundles (defined by representations of the fundamental group of $M$ ) are isospectral. In contrast, Gilkey [Gi] showed that some strongly isospectral spherical space forms constructed by A. Ikeda are not strongly $\pi_{1}$-isospectral.

For this reason, we next consider non-flat Hermitian line bundles $E$ over the manifold $M$. Associated to any metric on $M$ and metric connection $\nabla$ on $E$ is

Received August 2, 1996

1991 Mathematics Subject Classification 58G25, 22E30, 22E25.

Key words and phrases. Line bundles, Laplacian, isospectral deformations, nilmanifolds.

The first author is partially supported by a grant from the National Science Foundation. The third author was supported by a grant from DFG, Bonn/Germany and partially by SFB 256, Bonn. 
a bundle Laplacian with discrete spectrum. If one is given a trivial deformation $g_{t}$ of the metric on $M$-i.e., the $g_{t}$ are pairwise isometric - then one can always deform the connection through a family $\nabla_{t}$ so that the bundle Laplacians associated with $\left(g_{t}, \nabla_{t}\right)$ have spectrum independent of $t$. (In general, even in this case of a trivial deformation of the base metric, one must deform the connection along with the metric in order to keep the line bundle Laplacians isospectral.) Thus to spectrally detect the non-triviality of the isospectral deformations $\left(M, g_{t}\right)$, it suffices to find a Hermitian line bundle $E$ over $M$ and a connection $\nabla$ such that no choice of metric connections $\nabla_{t}$ with $\nabla_{0}=\nabla$ leads to isospectral bundle Laplacians on $E$.

This program was initiated by the second author in his thesis [Ou], where he considered several explicit examples. The third author [Sch] recently showed that the non-triviality of isospectral deformations of generic two-step nilmanifolds can be detected in this way. We describe the key ideas in section three.

The third author would like to thank Dartmouth College for its hospitality during her one year stay there.

\section{$\S 1$ Isospectral deformations}

A Riemannian nilmanifold is a compact quotient $\Gamma \backslash N$ of a simply-connected nilpotent Lie group $N$ by a discrete subgroup $\Gamma$ together with a Riemannian metric $g$ whose lift to $N$, again denoted $g$, is left-invariant. We will for simplicity refer to $g$ as a left-invariant metric on $\Gamma \backslash N$ even though $\Gamma \backslash N$ does not admit a left $G$-action.

The cocompact, discrete subgroup $\Gamma$ defines a rational structure on the Lie algebra $\mathcal{N}$ of $N$ as follows: The Lie group exponential map exp $: \mathcal{N} \rightarrow N$ is a diffeomorphism; we denote its inverse by $\log$. Then $\operatorname{span}_{\mathbb{Z}}(\log \Gamma)$ is a lattice of full rank in $\mathcal{N}$, each of whose elements is a rational multiple of an element of $\log \Gamma$; the structure coefficients with respect to any basis of this lattice are rational. (See $[\mathrm{Ra}]$ for these facts.) We call $X \in \mathcal{N}$ a $\Gamma$-rational vector if $X \in \operatorname{span}_{\mathbb{Q}}(\log \Gamma)$, and a linear subspace of $\mathcal{N}$ is called $\Gamma$-rational if it is spanned by $\Gamma$-rational vectors, or equivalently, if it is spanned by elements of $\log \Gamma$.

1.1 Definition. (i) An automorphism $\Phi$ of $N$ is said to be $\Gamma$-almost inner if $\Phi(\gamma)$ is conjugate in $N$ to $\gamma$ for every $\gamma \in \Gamma$. We denote the group of all $\Gamma$-almost inner automorphisms of $N$ by $\operatorname{AIA}(N ; \Gamma)$. A derivation $\varphi$ is said to be $\Gamma$-almost inner if $\varphi(X) \in[X, \mathcal{N}]$ for every $\Gamma$-rational vector $X \in \mathcal{N}$. The set of $\Gamma$-almost inner derivations forms a Lie algebra which we denote by $\operatorname{AID}(\mathcal{N} ; \Gamma)$.

(ii) An automorphism $\Phi$ of $N$, respectively a derivation $\varphi$ of $\mathcal{N}$, is called p-fold $\Gamma$-almost inner if for every $p$-dimensional $\Gamma$-rational subspace $\mathcal{U} \subseteq \mathcal{N}$ there exists $a \in N$ such that $\left.\Phi_{*}\right|_{\mathcal{U}}=\left.\operatorname{Ad}_{a}\right|_{\mathcal{U}}$, respectively, there exists $A \in \mathcal{N}$ such that $\left.\varphi\right|_{\mathcal{U}}=\left.\operatorname{ad}_{A}\right|_{\mathcal{U}}$.

According to [Go], $\operatorname{AIA}(N ; \Gamma)$ is a Lie subgroup of $\operatorname{Aut}(N)$, contained in the subgroup of unipotent automorphisms, and its Lie algebra is $\operatorname{AID}(\mathcal{N} ; \Gamma)$. It is easy to see that the same holds for the $p$-fold $\Gamma$-almost inner automorphisms and 
derivations. The group $\operatorname{Inn}(N)$ of inner automorphisms and, for each $p$, the group of $p$-fold $\Gamma$-almost inner automorphisms are clearly contained in $\operatorname{AIA}(N ; \Gamma)$; in general, these inclusions are nontrivial.

1.2 Theorem ([GW], [DG2], [Go]). If $\Phi \in \operatorname{AIA}(N ; \Gamma)$ and $g$ is left-invariant, then the Riemannian nilmanifolds $(\Gamma \backslash N, g)$ and $\left(\Gamma \backslash N, \Phi^{*} g\right)$ are isospectral.

In particular, if $\left\{\Phi_{t}\right\}$ is a continuous family of automorphisms in $\operatorname{AIA}(N ; \Gamma)$ with $\Phi_{0}=\mathrm{Id}$, then $\left(\Gamma \backslash N, \Phi_{t}^{*} g\right)$ is an isospectral deformation. As shown in [GW], $\left(\Gamma \backslash N, \Phi_{t}^{*} g\right)$ is a trivial deformation (i.e., the metrics are pairwise isometric) if and only if $\Phi_{t} \in \operatorname{Inn}(N)$ for every $t$.

In $[\mathrm{GW}$, a version of Theorem 1.2 is proved by representation theoretic techniques. A second proof is given in [DG2], under somewhat more general conditions, using heat trace methods. The latter proof follows the ideas of T. Sunada $[\mathrm{Su}]$. Both of these proofs show that the manifolds of Theorem 1.2 are strongly isospectral.

In the case of two-step nilmanifolds, a converse to Theorem 1.2 holds:

1.3 Theorem ([Pe], $[\mathrm{Ou}],[\mathrm{OP}])$. Let $N$ be a simply-connected two-step nilpotent Lie group, $\Gamma$ a cocompact, discrete subgroup of $N$, and $g_{t}$ a continuous family of left-invariant metrics such that $\left(\Gamma \backslash N, g_{t}\right)$ is an isospectral deformation. Then there exists a continuous family $\Phi_{t}$ of $\Gamma$-almost inner automorphisms of $N$ with $\Phi_{0}=\operatorname{Id}$ and $g_{t}=\Phi_{t}^{*} g_{0}$.

Theorem 1.3 fails for higher-step nilmanifolds as recent examples of R. Gornet [Gt] show.

\section{$\S 2$ Strong $\pi_{1}$-isospectrality}

Let $(\Gamma \backslash N, g)$ be a compact Riemannian nilmanifold. Any finite dimensional unitary representation $\sigma: \Gamma \rightarrow U(n)$ gives rise to a locally flat $n$-plane bundle $E_{\sigma}$ over $\Gamma \backslash N$. The bundle $E_{\sigma}$ may be identified with $N \times_{\sigma} \mathbb{C}^{n}$, i.e., the quotient of $N \times \mathbb{C}^{n}$ by the equivalence $(\gamma x, \sigma(\gamma) z) \sim(x, z)$ for $\gamma \in \Gamma, x \in N$, and $z \in \mathbb{C}^{n}$. The space $\mathcal{E}_{\sigma}$ of smooth sections of $E_{\sigma}$ is then identified with the space $C_{\sigma}(N)$ of all smooth $\mathbb{C}^{n}$-valued functions on $N$ satisfying $f(\gamma x)=\sigma(\gamma) f(x)$ for all $\gamma \in \Gamma$. The Riemannian metric $g$ on $\Gamma \backslash N$ defines a Laplace operator $\Delta^{\sigma}$ acting on $\mathcal{E}_{\sigma}$. Under the identification of $\mathcal{E}_{\sigma}$ with $C_{\sigma}(N)$, this Laplace operator coincides with the restriction to $C_{\sigma}(N)$ of the Laplacian $\Delta$ of $(N, g)$. (Note that $C_{\sigma}(N)$ is invariant under $\Delta$ since the left action of $\Gamma$ on $N$ is by isometries.)

2.1 Definition. Two left-invariant metrics $g_{1}$ and $g_{2}$ on $\Gamma \backslash N$ are said to be $\pi_{1}$-isospectral if for every finite dimensional unitary representation $\sigma$ of $\Gamma$, the associated bundle Laplacians $\Delta_{1}^{\sigma}$ and $\Delta_{2}^{\sigma}$ are isospectral.

2.2 Theorem. Let $\Phi$ be a $\Gamma$-almost inner automorphism of $N$. Then for any left-invariant metric $g$ on $N$, the metrics $g$ and $\Phi^{*} g$ are $\pi_{1}$-isospectral. Thus the isospectral deformations described in section one are also $\pi_{1}$-isospectral. 
2.3 Remark. As discussed in the introduction, the metrics are actually strongly $\pi_{1}$-isospectral. In fact, the proof below goes through when the bundle Laplacian is replaced by any natural self-adjoint elliptic operator with coefficients in $E_{\sigma}$.

Each of the two proofs of Theorem 1.2 referred to in section one can be modified to prove Theorem 2.2. The first proof is based on the Kirillov theory of representations of nilpotent Lie groups. In order to avoid an excursion into this theory, we outline only the second proof here.

We recall the notion of the heat kernel, first for the Laplace-Beltrami operator on a Riemannian manifold and then for bundle Laplacians. Let $M$ be a Riemannian manifold. A function $K: \mathbb{R}^{+} \times M \times M \rightarrow M$ is called a heat kernel, or fundamental solution of the heat equation, if it satisfies the following properties:

(K1) $K(t, x, y)$ is $C^{1}$ in $t$ and $C^{2}$ in $(x, y)$;

(K2) $\left(\frac{\partial}{\partial t}+\Delta_{x}\right) K(t, x, y)=0$ where $\Delta_{x}$ denotes the Laplacian acting on the second variable;

(K3) $\lim _{t \rightarrow 0^{+}} \int_{M} K(t, x, y) f(y) d y=f(x)$ for any smooth function $f$ with compact support on $M$.

In case $M$ is noncompact, $K$ is also required to satisfy a certain decay condition (see e.g. [Do].)

For compact Riemannian manifolds $M$, the trace of the heat kernel, defined by $Z(t)=\int_{M} K(t, x, x) d x$, satisfies $Z(t)=\sum_{j=0}^{\infty} e^{-\lambda_{j} t}$ where $\lambda_{0} \leq \lambda_{1} \leq \lambda_{2} \leq \ldots$ is the Laplace spectrum of $M$. Thus to show that two compact Riemannian manifolds are isospectral, it suffices to show that their heat kernels have the same trace.

The existence and uniqueness of the heat kernel on compact Riemannian manifolds is classical. H. Donnelly [Do] proved further that if $M$ is a possibly non-compact Riemannian manifold which covers a compact Riemannian manifold $\Gamma \backslash M$, then $M$ has a unique heat kernel $K$. Moreover, the heat kernel $K_{\Gamma}$ of $\Gamma \backslash M$ satisfies

$$
K_{\Gamma}(t, \bar{x}, \bar{y})=\sum_{\gamma \in \Gamma} K(t, x, \gamma(y))
$$

where $x$ and $y$ are any lifts of $\bar{x}$ and $\bar{y}$.

From the uniqueness of the heat kernel, one sees that

$$
K(t, a(x), a(y))=K(t, x, y) \text { for } a \in \operatorname{Iso}(M) .
$$

In the context of a Hermitian bundle $E$ over a Riemannian manifold $M$ with a metric connection, there is a similar notion of heat kernel $K$ (see [BGV]). Consider the bundle $E \otimes E^{*}$ over $M \times M$ given by $\bigcup_{(x, y) \in M \times M} \operatorname{Hom}\left(E_{y}, E_{x}\right)$. The heat kernel associated with the bundle Laplacian is a map $K: \mathbb{R}^{+} \times M \times M \rightarrow$ $E \otimes E^{*}$ which for each $t$ is a section of $E \otimes E^{*}$. The defining properties are analogous to (K1-3) above, with $f$ in (K3) being a section of $E$. 
Proof of Theorem 2.2. Let $K$ be the heat kernel associated with the LaplaceBeltrami operator of $(N, g)$. The heat kernel $K^{*}$ of $\left(N, \Phi^{*} g\right)$ is given by

$$
K^{*}(t, x, y)=K(t, \Phi(x), \Phi(y))
$$

Given a representation $\sigma: \Gamma \rightarrow U(n)$, a section $s$ of the bundle $E_{\sigma} \otimes E_{\sigma}^{*}$ corresponds to a function $F_{s}: N \times N \rightarrow \operatorname{End}\left(\mathbb{C}^{n}\right)$ satisfying

$$
F_{s}\left(\gamma_{1} x, \gamma_{2} y\right)=\sigma\left(\gamma_{1}\right) F_{s}(x, y) \sigma\left(\gamma_{2}^{-1}\right)
$$

for all $\gamma_{i} \in \Gamma, x, y \in N$. The correspondence is explicitly given by

$$
s(\bar{x}, \bar{y})=p_{x} \circ F_{s}(x, y) \circ p_{y}^{-1}
$$

for $\bar{x}, \bar{y} \in \Gamma \backslash N$, where $x$ and $y$ are any lifts to $N$ of $\bar{x}$ and $\bar{y}$, and $p_{x}$ denotes the restriction to the fiber over $x \in N$ of the projection $p: N \times \mathbb{C}^{n} \rightarrow N \times{ }_{\sigma} \mathbb{C}^{n} \cong E_{\sigma}$.

Denote by $K_{\sigma}$ the heat kernel for the bundle Laplacian $\Delta^{\sigma}$ on $\mathcal{E}_{\sigma} \cong C_{\sigma}(N)$. An argument similar to that in [Do] shows that the section $K_{\sigma}(t, \cdot, \cdot)$ of $E_{\sigma} \otimes E_{\sigma}^{*}$ corresponds (in the above sense) to the function

$$
(x, y) \mapsto \sum_{\gamma \in \Gamma} K(t, x, \gamma y) \sigma(\gamma)
$$

The fact that this function defines a section of $E_{\sigma} \otimes E_{\sigma}^{*}$, i.e., that it satisfies equation (3), is an elementary consequence of equation (1) and the fact that $\Gamma$ acts by isometries on $N$.

For $\gamma \in \Gamma$, we have $\Phi(\gamma)=a^{-1} \gamma a$ for some $a \in N$ and

$$
K^{*}(t, x, \gamma x)=K(t, \Phi(x), \Phi(\gamma x))=K\left(t, \Phi(x), a^{-1} \gamma a \Phi(x)\right)=K(t, a \Phi(x), \gamma a \Phi(x))
$$

by (1) and (2). Now the $\Gamma$-almost inner automorphism $\Phi$ is a volume preserving diffeomorphism of $N$; in fact its differential is a unipotent automorphism of the Lie algebra (see [DG2]). Since the isometry $a$ is also volume preserving, we would thus have $\int_{N} K^{*}(t, x, \gamma x) d x=\int_{N} K(t, x, \gamma x) d x$ if these integrals made sense. If also $\Gamma$ were finite, (4) would then show that $K_{\sigma}$ and $K_{\sigma}^{*}$ have the same trace. Of course the integrals above don't make sense and $\Gamma$ is infinite, but these complications can be handled in the same way as in the proof given in [DG2] of Theorem 1.2.

\section{$\S 3$ Spectra of line bundle Laplacians}

Let $(M, g)$ be a compact Riemannian manifold, and let $\pi: E \rightarrow M$ be a complex line bundle over $M$ endowed with a smooth Hermitian fiber metric $\langle.,$.$\rangle . Let P:=\{u \in E \mid\langle u, u\rangle=1\} \subset E$. Then $P$ can be considered as a 
principal $S^{1}$-bundle with which $E$ is associated via the canonical representation $S^{1} \rightarrow U(1)$.

The space $\mathcal{E}(E)$ of smooth sections of $E$ can be canonically identified with the space $C(P)^{S^{1}}$ of functions on $P$ given by

$$
C(P)^{S^{1}}:=\left\{f \in C^{\infty}(P, \mathbb{C}) \mid f(u z)=z^{-1} \cdot f(u) \forall u \in P, \forall z \in S^{1}\right\} .
$$

There is a canonical correspondence between connections on $P$ (that is, $S^{1}$-invariant distributions on $P$ which are complementary to the fiber direction) and metric connections on $E$. Consider a given connection $\mathcal{H}$ on $P$ and the corresponding metric connection $\nabla$ on $E$. For a smooth vector field $X$ on $M$, denote the unique $\mathcal{H}$-horizontal lift of $X$ to $P$ by $X^{*}$. The bundle Laplacian $\nabla^{*} \nabla: \mathcal{E}(E) \rightarrow \mathcal{E}(E)$ is given by

$$
\left(\nabla^{*} \nabla \Psi\right)(p)=-\sum_{i=1}^{n}\left(\nabla_{e_{i}(p)} \nabla_{e_{i}} \Psi-\nabla_{\widetilde{\nabla}_{e_{i}(p)} e_{i}} \Psi\right)
$$

where $\widetilde{\nabla}$ is the Levi-Cività connection associated with the metric $g$ on $M$, and $e_{1}, \ldots, e_{n}$ are vector fields in some neighborhood of $p$ such that $\left\{e_{1}(p), \ldots, e_{n}(p)\right\}$ is an orthonormal basis of $T_{p} M$. It is well-known that $\nabla^{*} \nabla$ is an elliptic selfadjoint positively semi-definite operator with discrete spectrum consisting of real nonnegative eigenvalues tending to infinity. Each eigenvalue occurs with finite multiplicity, and every eigenvector is a smooth section of $E$. Denote the spectrum of $\nabla^{*} \nabla$ by $\operatorname{spec}(g, \mathcal{H})$. Under the identification of $\mathcal{E}(E)$ with $C(P)^{S^{1}}$, the bundle Laplacian corresponds to the operator $\tilde{\Delta}: C(P)^{S^{1}} \rightarrow C(P)^{S^{1}}$ given by

$$
(\tilde{\Delta} f)(u)=-\sum_{i=1}^{n}\left(\left.e_{i}^{*}\right|_{u}\left(e_{i}^{*}(f)\right)-\left.\left(\widetilde{\nabla}_{e_{i}} e_{i}\right)^{*}\right|_{u}(f)\right),
$$

where $e_{1}, \ldots, e_{n}$ are given as in the definition of $\nabla^{*} \nabla$ (with $p:=\pi(u)$ ).

3.1 Remark. Consider the unique Riemannian metric $\tilde{g}$ on $P$ such that $\pi$ : $(P, \tilde{g}) \rightarrow(M, g)$ is a Riemannian submersion, the fibers of $P$ are $\tilde{g}$-orthogonal to the $\mathcal{H}$-horizontal subspaces, $\tilde{g}$ is $S^{1}$-invariant, and the fibers of $P$ have length 1. Let $W$ be the $S^{1}$-invariant unit vector field tangent to the fibers of $P$; i.e., for $u \in P$ we have $W(u)=\left.\frac{d}{d s}\right|_{s=0} e^{2 \pi i s} u$. Denote the Laplacian of the Riemannian manifold $(P, \tilde{g})$ by $\Delta_{\tilde{g}}$. Then $\tilde{\Delta}(f)=\Delta_{\tilde{g}}(f)+W^{2}(f)=\Delta_{\tilde{g}}(f)-4 \pi^{2} f$ for all $f \in C(P)^{S^{1}}$; thus

$$
\tilde{\Delta}=\left.\left(\Delta_{\tilde{g}}-4 \pi^{2} \mathrm{Id}\right)\right|_{C(P)^{S^{1}}}
$$

We now let $M=\Gamma \backslash N$ be a compact nilmanifold. A principal $S^{1}$-bundle $P$ over $N$ is isomorphic to a bundle of the form $\pi: \tilde{\Gamma} \backslash \tilde{N} \rightarrow \Gamma \backslash N$, where $\tilde{N}$ is 
a simply-connected one-dimensional central extension of $N, \tilde{\Gamma}$ is a cocompact, discrete subgroup of $\tilde{N}$ with $\tilde{\pi}(\tilde{\Gamma})=\Gamma$ where $\tilde{\pi}: \tilde{N} \rightarrow N$ is the homomorphic projection, and the bundle projection $\pi$ is induced by the homomorphism $\tilde{\pi}$. The Lie group $\tilde{N}$ is also nilpotent, and $\tilde{N}$ and $\tilde{\Gamma}$ are determined uniquely up to isomorphism. We will refer to $\tilde{\Gamma} \backslash \tilde{N}$ as a nilmanifold extension of $\Gamma \backslash N$. A connection $\mathcal{H}$ on $\tilde{\Gamma} \backslash \tilde{N}$, viewed as a distribution, will be called left-invariant if it lifts to a left-invariant distribution on $\tilde{N}$. Thus a left-invariant connection $\mathcal{H}$ is defined by a choice of vector space complement to the kernel of $\pi_{*}$ in the Lie algebra of $\tilde{N}$.

We will say an automorphism $\tilde{\Phi}$ of $\tilde{N}$ is a lift of an automorphism $\Phi$ of $N$ if $\tilde{\pi} \circ \tilde{\Phi}=\Phi \circ \tilde{\pi}$. Lifts to $\tilde{\mathcal{N}}$ of derivations on $\mathcal{N}$ are similarly defined.

3.2 Proposition. Let $\Gamma$ be a cocompact, discrete subgroup of a simply-connected nilpotent Lie group $N$ and let $\Phi_{t}$ be a continuous family of $\Gamma$-almost inner automorphisms of $N$. Let $g$ be a left-invariant metric on $N$ and set $g_{t}=\Phi_{t}^{*}(g)$. (Thus, by Theorem 1.2, the Riemannian nilmanifolds $\left(\Gamma \backslash N, g_{t}\right)$ are mutually isospectral.) Let $\tilde{\Gamma} \backslash \tilde{N}$ be a principal $S^{1}$-bundle over $\Gamma \backslash N$ and $E$ the line bundle associated with $\tilde{\Gamma} \backslash \tilde{N}$ by the canonical representation $S^{1} \rightarrow U(1)$. Let $\mathcal{H}$ be a leftinvariant connection on $\tilde{\Gamma} \backslash \tilde{N}$. Suppose that the $\Phi_{t}$ lift to a continuous family of $\tilde{\Gamma}$-almost inner automorphisms $\tilde{\Phi}_{t}$ of $\tilde{N}$. Set $\mathcal{H}_{t}=\left(\tilde{\Phi}_{t}\right)_{*}^{-1}(\mathcal{H})$. Then the bundle Laplacians $\tilde{\Delta}_{t}$ associated with the metrics $g_{t}$ on $\Gamma \backslash N$ and the connections $\mathcal{H}_{t}$ are mutually isospectral; i.e., $\operatorname{spec}\left(g_{t}, \mathcal{H}_{t}\right)$ is independent of $t$.

Proof. As in Remark 3.1, the pair $\left(g_{t}, \mathcal{H}_{t}\right)$ gives rise to a Riemannian metric $\tilde{g}_{t}$ on $\tilde{\Gamma} \backslash \tilde{N}$ for each $t$. These metrics are left-invariant and satisfy $\tilde{g}_{t}=\tilde{\Phi}_{t}^{*}(\tilde{g})$ where $\tilde{g}=$ $\tilde{g}_{0}$. Theorem 1.2 implies that the manifolds $\left(\tilde{\Gamma} \backslash \tilde{N}, \tilde{g}_{t}\right)$ are mutually isospectral. By the discreteness of $\operatorname{spec}\left(\tilde{\Gamma} \backslash \tilde{N}, \tilde{g}_{t}\right)$ and the continuity of the eigenvalues as $t$ varies, the Laplacians restricted to $C(\tilde{\Gamma} \backslash \tilde{N})^{S^{1}}$ are also isospectral. It then follows from equation (5) that the bundle Laplacians $\tilde{\Delta}_{t}$ are isospectral.

3.3 Remarks. (i) Even in the case where the $\Phi_{t}$ are inner automorphisms, so that the manifolds $\left(\Gamma \backslash N, g_{t}\right)$ are mutually isometric, it is still necessary to deform the connection as in the Proposition in order for the bundle Laplacians to be isospectral. Of course, in this case, it is always possible to deform the connection through left-invariant connections in order to obtain isospectrality. Thus in order to use line bundle Laplacians to prove the non-triviality of a deformation $g_{t}$ of left-invariant metrics on a given nilmanifold, one must find a line bundle and (left-invariant) connection so that no matter how one deforms the connection (through left-invariant connections), the spectrum of the line bundle Laplacian deforms non-trivially with $t$. (The assumption of left-invariance of the connections can be reformulated into a more intrinsic notion. See Remark 3.7 below.)

(ii) Let $\left\{\Phi_{t}\right\}_{t}$ be a one-parameter group of $\Gamma$-almost inner automorphisms of $N$, and let $\varphi \in \operatorname{AID}(\mathcal{N} ; \Gamma)$ be the generator of $\left\{\Phi_{t}\right\}_{t}$, i.e., $\Phi_{t *}=\exp t \varphi$. Then the $\Phi_{t}$ lift to $\tilde{\Gamma}$-almost inner automorphisms $\tilde{\Phi}_{t}$ of $\tilde{N}$ if and only if $\varphi$ lifts to a 
$\Gamma$-almost inner derivation $\tilde{\varphi}$ of $\mathcal{N}$.

Consider the following example of an isospectral deformation of a two-step nilmanifold, which was originally given in [GW]:

Let $\mathcal{N}$ be the Lie algebra with basis $\left\{X_{1}, Y_{1}, X_{2}, Y_{2}, Z_{1}, Z_{2}\right\}$ whose non-zero Lie brackets are given by

$$
\left[X_{1}, Y_{1}\right]=\left[X_{2}, Y_{2}\right]=Z_{1}, \quad\left[X_{1}, Y_{2}\right]=Z_{2} .
$$

Let $N$ be the simply connected Lie group with Lie algebra $\mathcal{N}$, and let $\Gamma$ be the cocompact, discrete subgroup of $N$ generated by the set $\exp \left\{X_{1}, Y_{1}, X_{2}, Y_{2}, Z_{1}, Z_{2}\right\}$. Consider the left-invariant metric $g$ on $N$ with respect to which the left-invariant vector fields $X_{1}, Y_{1}, X_{2}, Y_{2}, Z_{1}, Z_{2}$ are orthonormal. Let $\varphi$ be the derivation of $\mathcal{N}$ which maps $Y_{2}$ to $Z_{2}$ and the other basis elements to zero. Then $\varphi$ is almost inner: For $X=x_{1} X_{1}+y_{1} Y_{1}+x_{2} X_{2}+$ $y_{1} Y_{1}+y_{2} Y_{2}+z_{1} Z_{1}+z_{2} Z_{2}$ we have

$$
\varphi(X)= \begin{cases}{[0, X]} & \text { if } y_{2}=0 \\ {\left[X_{1}-\frac{y_{1}}{y_{2}} X_{2}, X\right]} & \text { if } y_{2} \neq 0 .\end{cases}
$$

Thus the one-parameter group of automorphisms $\Phi_{t}$ with differentials $\Phi_{t *}=$ $\exp t \varphi=\operatorname{Id}+t \varphi$ is a family of almost inner automorphisms of $N$, and $\left(\Gamma \backslash N, \Phi_{t}^{*} g\right)$ is an isospectral deformation. (See the remarks following Definition 1.1.) Each of the following examples concerns bundle Laplacians on some line bundle over this underlying isospectral deformation.

3.4 Example. Consider the central extension $\tilde{\mathcal{N}}$ of $\mathcal{N}$ with basis $\left\{\tilde{X}_{1}, \tilde{Y}_{1}, \ldots\right.$, $\left.\tilde{Z}_{2}, W\right\}$, whose nontrivial Lie brackets are given by

$$
\begin{gathered}
{\left[\tilde{X}_{1}, \tilde{Y}_{1}\right]=\left[\tilde{X}_{2}, \tilde{Y}_{2}\right]=\tilde{Z}_{1}, \quad\left[\tilde{X}_{1}, \tilde{Y}_{2}\right]=\tilde{Z}_{2}} \\
{\left[\tilde{X}_{1}, \tilde{X}_{2}\right]=W .}
\end{gathered}
$$

Let $\tilde{N}$ be the corresponding simply connected Lie group, and let $\tilde{\Gamma}$ be the subgroup of $\tilde{N}$ generated by the set $\exp \left\{\tilde{X}_{1}, \tilde{Y}_{1}, \ldots, \tilde{Z}_{2}, W\right\}$. Obviously $\tilde{\Gamma}$ is cocompact and discrete, and its projection to $N$ is $\Gamma$. Lift the derivation $\varphi$ to a derivation $\tilde{\varphi}$ of $\tilde{\mathcal{N}}$ given by

$$
\tilde{\varphi}\left(\tilde{Y}_{2}\right)=\tilde{Z}_{2}, \quad \tilde{\varphi}\left(\tilde{X}_{2}\right)=W,
$$

while the other basis elements of $\tilde{\mathcal{N}}$ are mapped to zero. This lift $\tilde{\varphi}$ is almost inner on $\tilde{\mathcal{N}}$ : For $X=x_{1} \tilde{X}_{1}+y_{1} \tilde{Y}_{1}+x_{2} \tilde{X}_{2}+y_{2} \tilde{Y}_{2}+z_{1} \tilde{Z}_{1}+z_{2} \tilde{Z}_{2}+w W$, we have

$$
\tilde{\varphi}(X)= \begin{cases}{[0, X]} & \text { if } x_{2}=y_{2}=0, \\ {\left[\tilde{X}_{1}-\frac{y_{1}}{y_{2}} \tilde{X}_{2}, X\right]} & \text { if } x_{1}=0, y_{2} \neq 0, \\ {\left[\tilde{X}_{1}+\frac{y_{1}}{x_{2}} \tilde{Y}_{2}, X\right]} & \text { if } x_{1}=0, x_{2} \neq 0, \\ {\left[-\frac{x_{2}}{x_{1}} \tilde{X}_{2}-\frac{y_{2}}{x_{1}} \tilde{Y}_{2}, X\right]} & \text { if } x_{1} \neq 0 .\end{cases}
$$


Let $\left\{\tilde{\Phi}_{t}\right\}$ be the one-parameter group of $\tilde{\Gamma}$-almost inner automorphisms generated by $\tilde{\varphi}$, so $\tilde{\Phi}_{t *}=\exp t \tilde{\varphi}=\operatorname{Id}+t \tilde{\varphi}$. By Proposition 3.2, the bundle Laplacians associated with the metrics $g_{t}$ on the base manifold and the nonconstant family of left-invariant connections

$$
\mathcal{H}_{t}:=\tilde{\Phi}_{t *}^{-1}(\mathcal{H})=\operatorname{span}\left\{\tilde{X}_{1}, \tilde{Y}_{1}, \tilde{X}_{2}-t W, \tilde{Y}_{2}-t \tilde{Z}_{2}, \tilde{Z}_{1}, \tilde{Z}_{2}, W\right\}
$$

are isospectral.

3.5 Example. Consider the extension $\tilde{\mathcal{N}}$ of $\mathcal{N}$ with basis $\left\{\tilde{X}_{1}, \tilde{Y}_{1}, \ldots, \tilde{Z}_{2}, W\right\}$, whose nontrivial Lie brackets are this time given by

$$
\begin{gathered}
{\left[\tilde{X}_{1}, \tilde{Y}_{1}\right]=\tilde{Z}_{1}+W, \quad\left[\tilde{X}_{2}, \tilde{Y}_{2}\right]=\tilde{Z}_{1},} \\
{\left[\tilde{X}_{1}, \tilde{Y}_{2}\right]=\tilde{Z}_{2}-W .}
\end{gathered}
$$

Let $\tilde{\Gamma}$ be the cocompact, discrete subgroup of the corresponding simply connected Lie group $\tilde{N}$ generated by the set $\exp \left\{\tilde{X}_{1}, \tilde{Y}_{1}, \ldots, \tilde{Z}_{2}, W\right\}$. In this example it turns out that there is no way to lift $\varphi$ to a $\tilde{\Gamma}$-almost inner derivation of $\tilde{\mathcal{N}}$ : Suppose $\tilde{\varphi}$ were such a lift. The conditions that $\tilde{\varphi}$ lifts $\varphi$ and that $\tilde{\varphi}\left(\tilde{Y}_{i}\right) \in\left[\tilde{\mathcal{N}}, \tilde{Y}_{i}\right]$ imply that $\tilde{\varphi}\left(\tilde{Y}_{1}\right)=0$ and $\tilde{\varphi}\left(\tilde{Y}_{2}\right)=\tilde{Z}_{2}-W$. But then $\tilde{\varphi}\left(\tilde{Y}_{1}+\tilde{Y}_{2}\right)=\tilde{Z}_{2}-W \notin\left[\tilde{\mathcal{N}}, \tilde{Y}_{1}+\tilde{Y}_{2}\right]=\operatorname{span}\left\{\tilde{Z}_{1}, \tilde{Z}_{2}\right\}$. Note that $\tilde{Y}_{1}, \tilde{Y}_{2}, \tilde{Y}_{1}+\tilde{Y}_{2}$ are $\tilde{\Gamma}$-rational vectors, hence $\tilde{\varphi}$ is not $\tilde{\Gamma}$-almost inner.

Thus (see Remark 3.3(ii)) one cannot lift $\Phi_{t}$ to a family of $\tilde{\Gamma}$-almost inner automorphisms of $\tilde{N}$. Since $\tilde{N}$ is still two-step nilpotent, it follows by Theorem 1.3 that for any continuous family of left-invariant connections $\mathcal{H}_{t}$ on $\tilde{\Gamma} \backslash \tilde{N}$, the Laplacian associated to the corresponding left-invariant metric $\tilde{g}_{t}$ on $\tilde{\Gamma} \backslash \tilde{N}$ has nonconstant spectrum. Analogously to the proof in [Sch, §3], one can show that even the spectrum of the restriction $\left.\Delta\right|_{C(\tilde{\Gamma} \backslash \tilde{N})^{S^{1}}}$ is nonconstant in $t$. Hence for every continuous family of left-invariant connections $\mathcal{H}_{t}$ on $\tilde{\Gamma} \backslash \tilde{N}$, the bundle Laplacians associated with $g_{t}$ and $\mathcal{H}_{t}$ will be non-isospectral.

The idea illustrated in Example 3.5 is developed in [Sch] to prove the following (recall Definition 1.1(ii)):

3.6 Theorem ([Sch, Theorem 1.7]). Let $N$ be a simply-connected two-step nilpotent Lie group, and let $\Gamma$ be a cocompact, discrete subgroup of $N$. Suppose that $N$ does not admit any 4-fold $\Gamma$-almost inner automorphisms that are not inner. Let $g_{t}$ be a continuous family of isospectral left-invariant metrics on $\Gamma \backslash N$. If the deformation $\left(\Gamma \backslash N, g_{t}\right)$ is nontrivial, then there exists a one-dimensional central extension $\tilde{N}$ of $N$ and a cocompact, discrete subgroup $\tilde{\Gamma}$ of $\tilde{N}$ whose projection to $N$ is $\Gamma$, such that for every continuous family of left-invariant connections $\mathcal{H}_{t}$ on the $S^{1}$-bundle $\pi: \tilde{\Gamma} \backslash \tilde{N} \rightarrow \Gamma \backslash N$, the spectrum $\operatorname{spec}\left(g_{t}, \mathcal{H}_{t}\right)$ is nonconstant in $t$.

We expect that the theorem is true without the technical hypothesis that $N$ does not admit any 4 -fold $\Gamma$-almost inner automorphisms. In any case, while 
it is easy to construct abundant examples of nilmanifolds $\Gamma \backslash N$ with $\Gamma$-almost inner, non-inner automorphisms, examples of such nilmanifolds admitting even 2 -fold $\Gamma$-almost inner, non-inner automorphisms are very rare.

3.7 Remark. There is also a more intrinsic version of this theorem (see [Sch, Theorem 1.15]) in which the statement is formulated in terms of a Hermitian line bundle $E$ over $\Gamma \backslash N$ and some intrinsically defined set $\mathcal{L}(E)$ of metric connections on $E$. Note that in the above formulation of the theorem, the set of left-invariant connections on $\tilde{\Gamma} \backslash \tilde{N}$ is not intrinsically defined in terms of just the bundle structure of $\tilde{\Gamma} \backslash \tilde{N}$, viewed as an $S^{1}$-bundle over $\Gamma \backslash N$, and the nilmanifold structure of the base manifold. The reason is that there are bundle isomorphisms from $\tilde{\Gamma} \backslash \tilde{N}$ to itself, inducing the identity on the base manifold, which do not preserve the nilmanifold structure of $\tilde{\Gamma} \backslash \tilde{N}$; in particular, they need not preserve the set of left-invariant connections on $\tilde{\Gamma} \backslash \tilde{N}$.

The set $\mathcal{L}(E)$ is defined as the set of all metric connections on $E$ for which the corresponding connection on the associated principal $S^{1}$-bundle $P$ corresponds to a left-invariant connection under some realization of $P$ as a nilmanifold extension $\tilde{\Gamma} \backslash \tilde{N}$ of $\Gamma \backslash N$, i.e., under a bundle isomorphism from $P$ to a nilmanifold extension $\tilde{\Gamma} \backslash \tilde{N}$ which induces the identity on $\Gamma \backslash N$.

Then the more intrinsic version of the above theorem asserts the existence of a Hermitian line bundle $E$ over $\Gamma \backslash N$ such that for every continuous family of metric connections $\nabla_{t} \in \mathcal{L}(E)$, the spectrum of the bundle Laplacians associated with $g_{t}$ and $\nabla_{t}$ is nonconstant in $t$. ("Continuous" is to be understood here with respect to some appropriate topology on $\mathcal{L}(E)$ which is defined in [Sch].) As shown in [Sch], the intrinsic version of the theorem actually turns out to be equivalent to the version cited above.

\section{References}

[BGV] N. Berline, E. Getzler, and M. Vergne, Heat kernels and Dirac operators, Springer, Berlin/Heidelberg/New York, 1991.

[DG1] D. DeTurck and C. Gordon, Isospectral deformations I: Riemannian structures on two-step nilspaces, Comm. Pure Appl. Math. 40 (1987), 367-387.

[DG2] , Isospectral Deformations II: Trace formulas, metrics, and potentials, Comm. Pure Appl. Math. 42 (1989), 1067-1095.

[Do] H. Donnelly, Asymptotic expansions for the compact quotients of group actions, Illinois J. Math. 23 (1979), 485-496.

[Gi] P. Gilkey, On spherical space forms with meta-cyclic fundamental group which are isospectral but not equivariant cobordant, Compositio Math. 56 (1985), 171-200.

[Go] C. Gordon, The Laplace spectra versus the length spectra of Riemannian manifolds, Contemp. Math. 51 (1986), 63-80.

[GW] C. Gordon, E. Wilson, Isospectral deformations of compact solvmanifolds, J. Diff. Geom. 19 (1984), 241-256.

[Gt] R. Gornet, Continuous families of Riemannian manifolds isospectral on functions but not on 1-forms, preprint.

[KN] S. Kobayashi, K. Nomizu, Foundations of differential geometry, Vol. 1, Interscience, New York, 1963. 
[OP] H. Ouyang, H. Pesce, Déformations isospectrales sur les nilvariétés de rang deux, C. R. Acad. Sci. Paris, Sér. I, 314 (1992), 621-623.

[Ou] H. Ouyang, On isospectral deformations on two-step nilmanifolds, Ph.D. thesis (1991), Washington University, St. Louis.

[Pe] H. Pesce, Calcul du spectre d'une nilvariété de rang deux et applications, Trans. Amer. Math. Soc. 339 (1993), 433-461.

[Ra] M. S. Raghunathan, Discrete subgroups of Lie groups, Springer, Berlin/New York, 1972.

[Sch] D. Schueth, Line bundle Laplacians over isospectral nilmanifolds, Trans. Amer. Math. Soc. (to appear).

[St] P. Stredder, Natural differential operators on Riemannian manifolds and representations of the orthogonal and special orthogonal group, J. Diff. Geom. 10 (1975), 647-660.

[Su] T. Sunada, Riemannian coverings and isospectral manifolds, Ann. of Math. 121 (1985), $169-186$.

Department of Mathematics, Dartmouth College, Hanover, NH 03755

E-mail address: Carolyn.S.Gordon@Dartmouth.edu

10834 Blaney Ave., Cupertino, CA 95014

E-mail address: ouyang@artsci.wustl.edu

Mathematisches Institut der Univ. Bonn, Beringstr. 1, D-53115 Bonn, GERMANY

E-mail address: schueth@math.uni-bonn.de 